\title{
In vitro characterization of stem/progenitor cells from semitendinosus and gracilis tendons as a possible new tool for cell-based therapy for tendon disorders
}

\author{
DEBORAH STANCO ${ }^{1}$, MARCO VIGANÒ ${ }^{1}$, CARLOTTA PERUCCA ORFEI ${ }^{1}$, ALESSIA DI GIANCAMILLO², \\ GABRIELE THIEBAT ${ }^{3}$, GIUSEPPE PERETTI ${ }^{4}$, LAURA DE GIROLAMO ${ }^{1}$ \\ ${ }^{1}$ Orthopaedic Biotechnology Laboratory, IRCCS Galeazzi Orthopaedic Institute, Milan, Italy \\ 2 Department of Health, Animal Science and Food Safety, University of Milan, Italy \\ ${ }^{3}$ Sport Traumatology and Arthroscopic Unit, IRCCS Galeazzi Orthopaedic Institute, Milan, Italy \\ ${ }^{4}$ Department of Biomedical Sciences for Health, University of Milan; IRCCS Galeazzi Orthopaedic Institute, Milan, Italy
}

\begin{abstract}
Purpose: this study was conducted to characterize tendon stem/progenitor cells (TSPCs) isolated from human semitendinosus and gracilis tendons in terms of stemness properties and multi-differentiation potential.

Methods: TSPCs were isolated from waste portions of semitendinosus and gracilis tendons from three donors who underwent anterior cruciate ligament reconstruction. TSPCs were plated in culture until passage 4, when experiments to assess cell proliferation, viability and clonogenic ability were performed. The immunophenotype of TSPCs was evaluated by cytofluorimetric analysis. The in vitro osteogenic, chondrogenic, adipogenic and tenogenic potential was evaluated using biochemical, histological and gene expression analysis to detect specific differentiation markers. Statistical analysis was performed using Student's t-test.

Results: after a few passages in culture the cell populations showed a homogeneous fibroblast-like morphology typical of mesenchymal stem cells. The average doubling time of TSPCs increased from $52.4 \pm 4.8$ at passage 2 to $100.8 \pm 23.4$ hours at passage 4 . The highest percentage of colonies was also found at passage $4(4.7 \pm 2.3 \%)$. TSPCs showed the typical mesenchymal phenotype, with high expression of CD73, CD90
\end{abstract}

\section{Corresponding Author:}

Laura de Girolamo, BS, PhD

Orthopaedic Biotechnology Laboratory

IRCCS Galeazzi Orthopaedic Institute

Via Riccardo Galeazzi 4, 20161 Milan, Italy

E-mail: laura.degirolamo@grupposandonato.it and CD105 and no expression of CD34 and CD45. Cells induced to differentiate toward osteogenic lineage showed significant upregulations of ALP activity $(+189 \%, \mathrm{p}<0.05)$ and calcified matrix deposition $(+49 \%, \mathrm{p}<0.05)$ compared with undifferentiated cells; culture in chondrogenic medium also provoked a significant increase in glycosaminoglycan levels $(+108 \%$, $\mathrm{p}<0.05)$. On the other hand, TSPCs were not able to respond to adipogenic stimuli. Scleraxis gene expression and decorin gene expression, considered tenogenic markers, were already very high in control cells, and culture in tenogenic medium further increased these values although not significantly.

Conclusions: our data show that it is possible to isolate TSPCs from very small fragments of tissue and that they show the typical features of MSCs and multi-differentiation potential, above all toward osteogenic and chondrogenic lineages.

Clinical relevance: this study can be considered one of the first attempts to clarify the biology of tendon cell populations, focusing in particular on the potential applicability of this cell source for future regenerative medicine purposes.

Key Words: tendon, stem/progenitor cells, multi-differentiation potential, tenogenic differentiation, tendon markers.

\section{Introduction}

Tendon injuries range from acute traumatic ruptures to chronic overuse and degenerative tendinopathies and the healing process is rarely able to restore tissue 
showing normal physiology. For this reason, despite improvements in therapeutic strategies (both conservative and surgical), outcomes often remain unsatisfactory $(1,2)$. The poor cellularity of tendon tissue is one of the main reasons for its limited regenerative capacity; indeed, tendon cells named tenocytes account for only $5 \%$ of the tissue and are responsible for the synthesis of all the extracellular matrix (ECM) components. For this reason, enrichment with an appropriate cell population through a regenerative medicine approach may enhance the healing response and prevent the degenerative process. Mesenchymal stem cells (MSCs), due to their availability, stable phenotype, low immunogenicity and ability to differentiate into several cell lineages according to environmental stimuli, are an ideal cell population for regenerative medicine and tissue engineering approaches. Although MSCs from different adult tissues have been extensively investigated (3-6), to date few in vivo and in vitro studies have considered the resident progenitor cells in tendon tissue. It was quite recently shown that tendon tissue contains not only tenocytes, but also a tendon stem/progenitor cell (TSPC) population $(7,8)$; this observation has allowed remarkable advances in understanding the physiopathology of this tissue, and also contributed to the search for a new potential tool for the regenerative treatment of tendon disorders.

In the orthopedic field the differentiation potential of MSCs into osteogenic and chondrogenic lineages has been extensively studied $(9,10)$; on the contrary few data concerning their tenogenic differentiation are available.

The purpose of the present study was to characterize in vitro TSPCs isolated from human hamstring tendons (semitendinosus and gracilis tendons) in term of their clonogenic and proliferation ability, immunophenotype profile and multi-lineage differentiation potential, including their ability to differentiate toward the tenogenic lineage. The hypothesis of the study was that TSPCs isolated from human hamstrings have multi-differentiation potential.

\section{Methods}

Isolation and culture of TSPCs and adipose-derived stem cells All the procedures were carried out at the Galeazzi Orthopaedic Institute, Milan, Italy with Institutional Review Board approval (M-SPER-014.ver 7 for use of surgical waste). TSPCs were isolated from discarded fragments of semitendinosus and gracilis tendons collected, under written consent, from three donors who underwent anterior cruciate ligament (ACL) reconstruction (Fig. 1). The tendon tissue samples were minced into small pieces $(0.5-1 \mathrm{~cm})$, placed in $100 \mathrm{~mm}$ Petri culture dishes and covered with control medium consisting of high glucose DMEM (HG-DMEM), $10 \%$ fetal bovine serum (FBS; Sigma-Aldrich, Saint Louis, MO, USA), $100 \mathrm{U} / \mathrm{ml}$ penicillin, $100 \mu \mathrm{g} / \mathrm{ml}$ streptomycin, $0.29 \mathrm{mg} / \mathrm{ml}$ L-glutamine (Life Technologies, Carlsbad, CA, USA). During the first 10

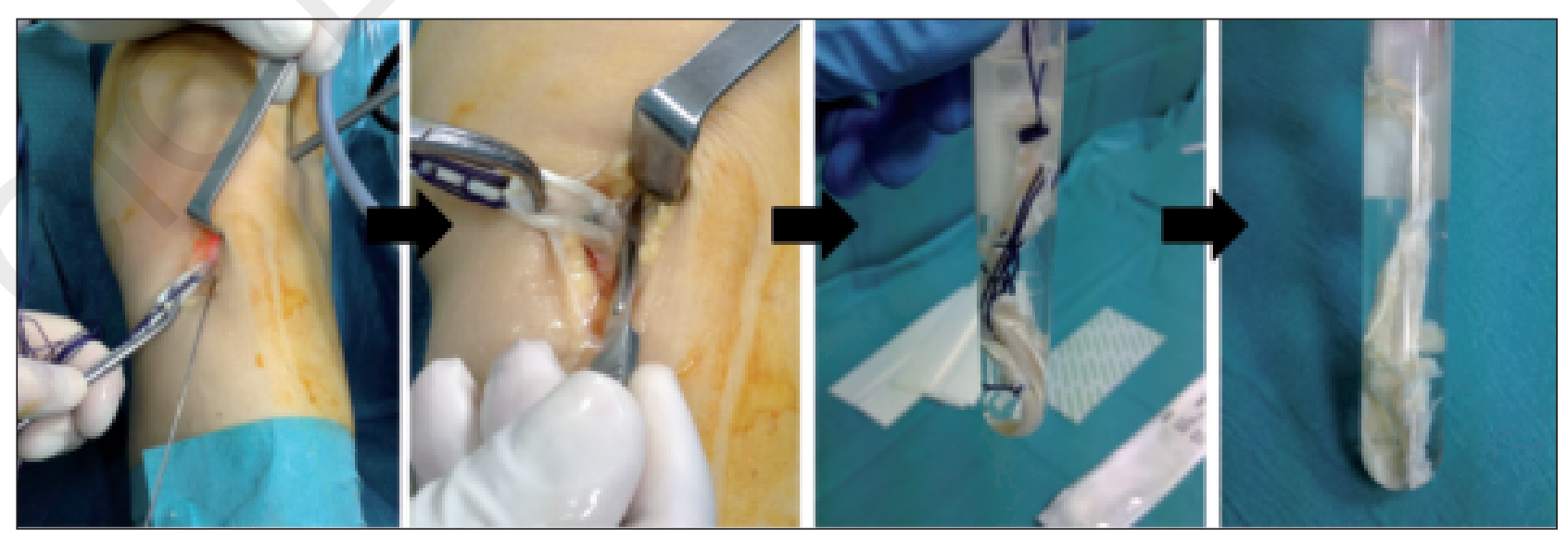

Fig. 1. Surgical harvesting of hamstring tendons; after the preparation of the pro-ACL graft, the remnant portions of both semitendinosus and gracilis tendons were collected and sent to the laboratory for cell isolation. 
days in culture, the tendon cells migrated out of the tissue and started to proliferate, reaching confluence after about three weeks. TSPCs were kept in culture, changing the culture medium every three days; when they reached $80-90 \%$ of confluence, cells were detached by incubation with trypsin/EDTA (0.5\% trypsin/0.2\% EDTA; Sigma-Aldrich) and plated at a density of $3 \mathrm{x}$ $10^{3} \mathrm{cells} / \mathrm{cm}^{2}$ for the further experiments.

\section{Proliferation ability}

Cell-doubling time (DT), an indicator of cell proliferation rate, was analyzed from passage 2 up to passage 4. DT was calculated according to the following formula:

\section{$\mathrm{DT}=\mathrm{CT} / \ln \left(\mathrm{N}_{\mathrm{f}} \mathrm{N}_{\mathrm{i}}\right) / \ln 2$}

where DT is the cell-doubling time, CT the cell culture time (hours), $\mathrm{N}_{\mathrm{f}}$ the final number of cells, and $\mathrm{N}_{\mathrm{i}}$ the initial number of cells.

\section{Cell viability}

Cell viability assay was performed at 1, 4 and 7 days of culture on cells at passage 4. Briefly, a final concentration of $0.5 \mathrm{mg} / \mathrm{mL}$ MTT [3-(4,5-dimethylthi azol-2yl)-2,5-diphenyltetrazolium bromide, Sigma-Aldrich] was added to the culture medium and incubated for four hours at $37^{\circ} \mathrm{C}$ in 96 -well plate $\left(1.5 \times 10^{4}\right.$ cells per well); the medium was removed and 100\% DMSO was added to each well to solubilize the precipitate. Absorbance was read at $570 \mathrm{~nm}$.

\section{Clonogenic ability}

Colony-forming unit fibroblast (CFU-F) assay was performed on TSPC populations from passage 2 to passage 4 as previously described (11). Briefly, the cells were plated at low density by limiting dilution (starting dilution: $100 \mathrm{cells} / \mathrm{cm}^{2}$, ending dilution: 15 cells $/ \mathrm{cm}^{2}$ ) and cultured in HG-DMEM supplemented with 20\% FBS for 14 days. The cells were then fixed with methanol, stained with crystal violet staining (Sigma-Aldrich) and then counted. The CFU-F frequency was calculated by scoring the individual colonies composed of at least 30 cells and expressed as a percentage of the initial number of seeded cells.

\section{Immunophenotype}

Cytofluorimetric analysis (fluorescence-activated cell sorting, FACS) was used to characterize the immuno- phenotype of the TSPCs. The cells were detached and washed twice in cold FACS buffer (PBS w/o $\mathrm{Ca} / \mathrm{Mg}^{2+}$ containing $2 \% \mathrm{FBS}$ and $\left.0.1 \% \mathrm{NaN}_{3}\right) .2 .5 \times 10^{5}$ cells were incubated with anti-human primary monoclonal antibodies raised against CD13, CD29, CD 31, CD34, CD44, CD45, CD54, CD71, CD73, CD90, CD105 and CD166 (MiltenyiBiotec, BergischGladbach, Germany; Ancell Corporation, Bayport, MN, USA). Where needed, streptavidin-PE and FITCconjugated goat anti-mouse Abs (Ancell Corporation) were used as secondary antibodies. Data were acquired by FACS Calibur flow cytometer and analyzed by Cell Quest software (BD Bioscences, San Jose, NJ, USA).

\section{Adipogenic differentiation}

Cells at passage 4 were induced to differentiate toward adipogenic lineage by using a repeated pulsed protocol (4) consisting of three days in adipogenic induction medium (control medium supplemented with $1 \mu \mathrm{M}$ dexamethasone, $10 \mu \mathrm{g} / \mathrm{mL}$ insulin, $500 \mu \mathrm{M}$ 3-isobutyl-1-methylxanthine and $200 \mu \mathrm{M}$ indomethacin), followed by three days in maintenance medium (control medium supplemented with $10 \mu \mathrm{g} / \mathrm{mL}$ insulin). The total duration of the differentiation treatment was 21 days. Lipid vacuoles were stained with Oil Red O (Sigma-Aldrich), then unstained with 100\% isopropanol. The absorbance of the resulting solution was read at $490 \mathrm{~nm}$ using a spectrophotometer (VictorX3, Perkin Elmer microplate, MA, USA).

\section{Osteogenic differentiation}

Cells at passage 4 were differentiated into osteogenic lineage in osteogenic medium (control medium supplemented with $10 \mathrm{mM}$ glycerol-2-phosphate, $10 \mathrm{nM}$ dexamethasone, $150 \mu \mathrm{M}$ l-ascorbic acid-2-phosphate and $10 \mathrm{nM}$ cholecalciferol) as previously described (12). After 14 days of differentiation, cells were assessed for alkaline phosphatase (ALP) activity which was determined by enzymatic assay incubating cellular lysates at $37^{\circ} \mathrm{C}$ with $1 \mathrm{mM}$ p-nitrophenyl phosphate in alkaline buffer $(100 \mathrm{mM}$ diethanolamine and 0.5 $\mathrm{mM} \mathrm{MgCl}_{2}, \mathrm{pH}$ 10.5). Absorbance was read at 405 $\mathrm{nm}$. ALP activity was then normalized for total protein content determined using a BCA protein assay kit (Pierce Biotechnology, Rockford, IL, USA).

Extracellular calcified matrix deposition was also evaluated after 21 days of differentiation. Briefly, cells were 
fixed with ice-cold $70 \%$ ethanol for $1 \mathrm{~h}$, and stained with $40 \mathrm{mM}$ Alizarin Red S (Fluka Sigma-Aldrich) ( $\mathrm{pH}$ 4.1) for $15 \mathrm{~min}$. The dye was extracted with $10 \%$ cetylpyridinium chloride monohydrate (CPC; SigmaAldrich) in $0.1 \mathrm{M}$ phosphate buffer $(\mathrm{pH} 7.0)$ and the absorbance of the resulting solution was read at $550 \mathrm{~nm}$.

\section{Chondrogenic differentiation}

The chondrogenic induction was performed using the so-called pellet-culture system, which is able to give cells a three-dimensional microenvironment that is known to promote the chondrogenic process (10) (Fig. 2). TSPCs $\left(5.0 \times 10^{5}\right)$ were centrifuged $(250 \mathrm{~g}, 5 \mathrm{~min})$ in centrifuge tubes to obtain cell pellets. Pellets were then cultured for 21 days in chondrogenic medium consisting of HG-DMEM supplemented with $100 \mathrm{U} / \mathrm{ml}$ penicillin, $100 \mu \mathrm{g} / \mathrm{ml}$ streptomycin, $0.29 \mathrm{mg} / \mathrm{ml} \mathrm{L}$-glutamine, $1 \mathrm{mM}$ sodium pyruvate, $1.25 \mathrm{mg} / \mathrm{ml}$ human serum albumin (HAS; Sigma-Aldrich), 1\% ITS+1 (containing $1.0 \mathrm{mg} / \mathrm{ml}$ insulin from bovine pancreas, 0.55 $\mathrm{mg} / \mathrm{ml}$ human transferrin, $0.5 \mu \mathrm{g} / \mathrm{ml}$ sodium selenite, $50 \mathrm{mg} / \mathrm{ml}$ bovine serum albumin and $470 \mu \mathrm{g} / \mathrm{ml} \mathrm{lino-}$ leic acid (Sigma-Aldrich), $0.1 \mu \mathrm{M}$ dexamethasone, 0.1 mM L-ascorbic acid-2-phosphate, and 10 ng/ml TGF$\beta 1$ (PeproTech, Rocky Hill, NJ, USA) (13). For histological analysis, pellets were fixed for $24 \mathrm{~h}$ in $10 \%$ neutral buffered formalin, embedded in paraffin and sectioned at $4 \mu \mathrm{m}$. Sections were stained with hematoxylineosin (Sigma-Aldrich) and safran in order to evaluate deposition of ECM and glycosaminoglycans (GAGs). For quantification of GAGs, pellets were digested (16 h, $\left.60^{\circ} \mathrm{C}\right)$ in $500 \mu \mathrm{l}$ of PBE buffer ( $100 \mathrm{mM} \mathrm{Na} 2 \mathrm{HPO} 4,10$ $\mathrm{mM} \mathrm{Na}$ EDTA, pH 6.8) containing $1.75 \mathrm{mg} / \mathrm{ml} \mathrm{L}$ cystein (Sigma-Aldrich) and $14.2 \mathrm{U} / \mathrm{ml}$ papain (Worthington, Lakewood,

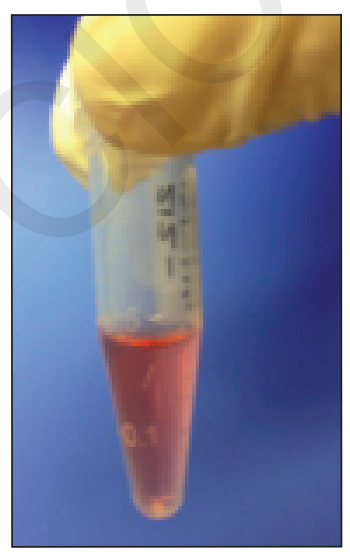
NJ, USA). Samples were incubated with $16 \mathrm{mg} / \mathrm{l}$ dimethylmethylene blue (SigmaAldrich) and absorbance was read at $500 \mathrm{~nm}$ (Perkin Elmer Victor X3 microplate reader). The same samples were used for DNA quantification,

Fig. 2. Cell micromass or pellet lying on the bottom of the centrifuge tube. Pellet-culture system was used to increase the chondrogenic differentiation of the cells as promoted by the three-dimensional croenvironment. expression of the cell proliferation rate, by CyQUANT Kit (Life Technologies). The amount of GAGs produced for each sample was then normalized for DNA content and expressed as $\mu \mathrm{g}$ of GAGs per $\mu \mathrm{g}$ of DNA.

\section{Tenogenic differentiation}

Cells at passage 4 were induced to differentiate toward tenogenic lineage for 14 days in inductive medium consisting of HG-DMEM supplemented with $1 \%$ FBS (Sigma-Aldrich), $100 \mathrm{U} / \mathrm{ml}$ penicillin, $100 \mu \mathrm{g} / \mathrm{ml}$ streptomycin, $0.29 \mathrm{mg} / \mathrm{ml} \mathrm{L}$-glutamine, and $50 \mathrm{ng} / \mathrm{ml}$ BMP-12 (PeproTech). Evaluation of the expression of tendon-related genes was assessed at 7 and 14 days by RT-PCR (14). Briefly, total RNA was purified from cell lysates (RNeasy Mini kit, Qiagen, Germany) and reverse-transcripted to cDNA using the iScriptc DNA Synthesis Kit (Bio-Rad Laboratories, CA, USA). 10 ng of cDNA were incubated with a PCR mix containing TaqMan Universal PCR Master Mix and Assays-onDemand Gene expression probes (Life Technologies) for the following genes: collagen type I alpha 1 (COL1A1), collagen type III alpha 1 (COL3A1), scleraxis $(S C X)$, decorin $(D C N)$. The reaction was performed with Applied Biosystems Step One Plus (Life Technologies). The fold change in the expression was normalized for the expression of the housekeeping gene glyceraldehyde-3-phosphate dehydrogenase (GAPDH).

\section{Statistical analysis}

Data are expressed as mean values \pm standard deviation (SD). Normal distribution of values was assessed by the Kolmogorov-Smirnov normality test. Statistical analysis was performed using Student's t-test for data with a normal distribution and the Wilcoxon test for data with a non-normal distribution (Graph Pad Prism v 5.00; Graph Pad Software, San Diego, CA, USA); $<<0.05$ was considered statistically significant.

\section{Results}

TSPCs were harvested from hamstring tendons of three donors as listed in Table 1 . The number of cells calculated at passage 1 was $6.4 \pm 0.4 \times 10^{5}$ per gram of tissue. During passages in culture, the cell population, initially heterogeneous and composed of both tenocytes and TSPCs, progressively became more homoge- 
nous, with a net prevalence of TSPCs (Fig. 3a), as terminally differentiated tenocytes are known to be unstable in culture and slower to grow. The TSPCs showed the fibroblastic-like morphology typical of these cells.

\section{Stemness features}

At passages 2 and 3 cells showed an average DT of 52.4 \pm 4.8 and $55.9 \pm 17.3$ hours, respectively, whilst at passage 4 the average DT was higher $(100.8 \pm 23.4$ hours $)$, although the difference with regard to the previous passages was not statistically significant (Fig. 3b). At passage 4 all the TSPC populations possessed similar viability characterized by a slightly increasing trend with time spent in culture (Fig. 3c). Similarly to the proliferation rate, the clonogenic ability of TSPCs increased with passages, with the highest value being observed at passage $4(4.7 \pm 2.3 \%)$, although this increase did not represent a statistically significant difference compared with the previous passages (Fig. 3d). At passage 4 the phenotype of TSPCs was homogeneous as a high percentage of them expressed the MSC-specific surface antigens CD13, CD44, CD73, CD90, CD29, CD105, CD166 and CD54. Moreover, they were negative for the hematopoietic cell markers CD34, CD31, CD71 and CD45 (Tab. 2).
Table 1. Donor and tissue characteristics of TSPCs.

\begin{tabular}{lllll}
\hline & $\begin{array}{l}\text { Gender } \\
(\mathrm{M} / \mathrm{F})\end{array}$ & $\begin{array}{l}\text { Age } \\
(\text { years })\end{array}$ & $\begin{array}{l}\text { Tissue weight } \\
(\mathrm{g})\end{array}$ & $\begin{array}{l}\mathrm{N}^{\circ} \text { cells/g } \\
\text { of tissue }\end{array}$ \\
\hline TSPC1 & $\mathrm{M}$ & 44 & 2.6 & $0.5 \times 10^{6}$ \\
TSPC2 & $\mathrm{M}$ & 20 & 3 & $0.3 \times 10^{6}$ \\
TSPC3 & $\mathrm{F}$ & 27 & 1.6 & $1.0 \times 10^{6}$ \\
\hline Mean \pm SD & $\mathbf{3 0 \pm 1 2}$ & $\mathbf{2 . 4 \pm 0 . 7}$ & $\mathbf{0 . 6 \pm 0 . 3 \times 1 0 ^ { 6 }}$ & \\
\hline
\end{tabular}

Table 2. Surface marker expression of TSPCs.

\begin{tabular}{lll}
\hline & Markers & Positive cells (\%) \\
\hline MSC markers & CD13 & $99.5 \pm 0.6$ \\
& CD44 & $99.1 \pm 1.0$ \\
& CD73 & $98.4 \pm 2.8$ \\
& CD90 & $92.4 \pm 6.4$ \\
& CD29 & $90.4 \pm 13.8$ \\
& CD105 & $86.4 \pm 11.3$ \\
& CD166 & $81.6 \pm 16.6$ \\
& CD54 & $74.2 \pm 11.3$ \\
\hline \multirow{2}{*}{ Hematopoietic markers } & CD34 & $6.1 \pm 6.5$ \\
& CD31 & $1.4 \pm 0.9$ \\
& CD71 & $1.4 \pm 0.7$ \\
& CD45 & $0.7 \pm 0.6$ \\
\hline
\end{tabular}

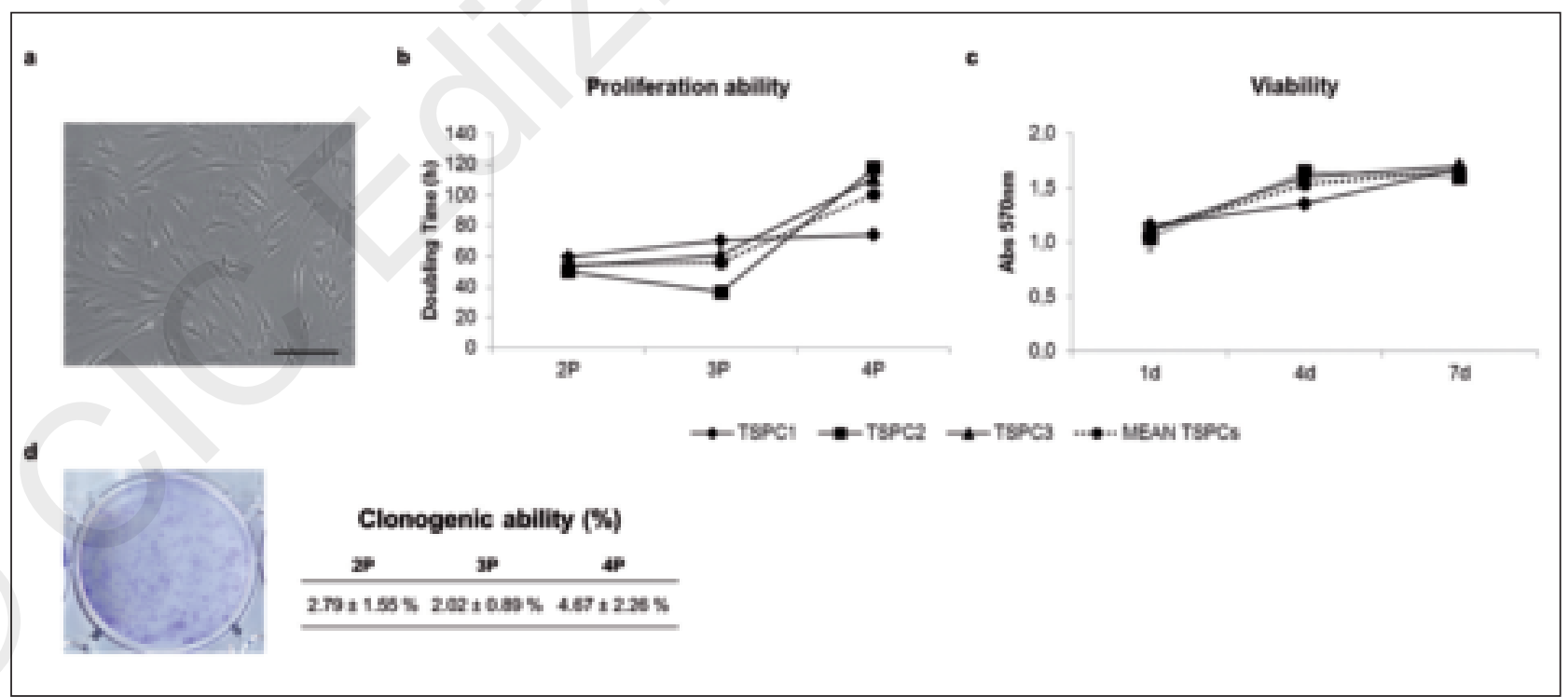

Fig. 3. a: Morphology of a representative TSPC population at passage 4 in culture. Scale bar=200 $\mu \mathrm{m}$; b: Proliferation ability of TSPCs from passage 2 to 4, expressed as doubling time (hours). Both proliferation trend of the single populations and the mean value are shown; c: Viability of TSPCs at passage 4 assessed by MTT assay at 1, 4 and 7 days. Both viability of the single populations and the mean value are shown; d: Representative cell sample stained with Crystal Violet to assess colony formation and relative mean( \pm standard deviation) clonogenic ability of TSPCs from passage 2 to passage 4 . 


\section{oints}

D. Stanco et al.

In vitro multi-differentiation potential

The in vitro multi-differentiation potential of TSPCs was also assessed. After 14 days of adipogenic induction, cells progressively lost their fibroblast-like morphology and started to produce intra-cytoplasmic lipid vacuoles, as shown by Oil Red O staining (Fig.4a). The dye extraction revealed a $57 \%$ increase in lipid vacuole content in differentiated cells in comparison with cells cultured in control medium, although this difference was not significant (Fig. 4b). On the other hand, TSPCs were able to differentiate toward the osteogenic lineage when cultured for 14 and 21 days in osteo-inductive medium, as shown by the significant upregulation of ALP activity $(+189 \%$; $\mathrm{p}<0.05)$ and calcified matrix deposition $(+49 \%, \mathrm{p}<0.05)$, compared with control cells (Fig. 5). After 21 days of chondrogenic induction in pellet culture conditions, histological evaluation revealed larger dimensions of the pellets cultured in chondrogenic medium as well as superior deposition of GAGs in differentiated TSPCs compared with controls (Fig. 6a). According to the histological results, a significantly higher amount of GAGs was measured by biochemical assay in TSPC pellets cultured in chondrogenic medium compared with control pellets $(+108 \%$; $<<0.05)$ (Fig. 6 b).

\section{a}

b
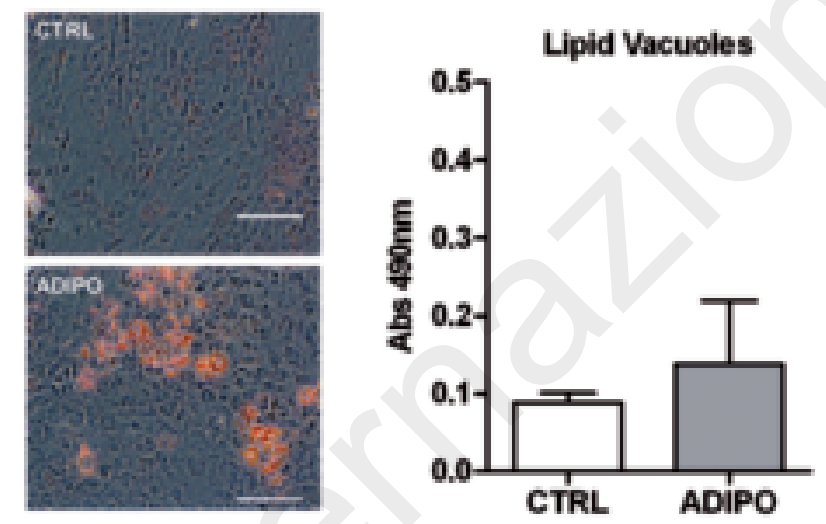

Fig. 4. a: Lipid vacuole production of a representative TSPC population cultured in control (CTRL) or in adipogenic (ADIPO) medium and stained with Oil Red 0 for 21 days. Scale bar $=200 \mu \mathrm{m}$; b: Quantification of the Oil Red O dye extraction from both control and adipogenic TSPCs. Data are expressed as mean values \pm standard deviation $(n=3)$.

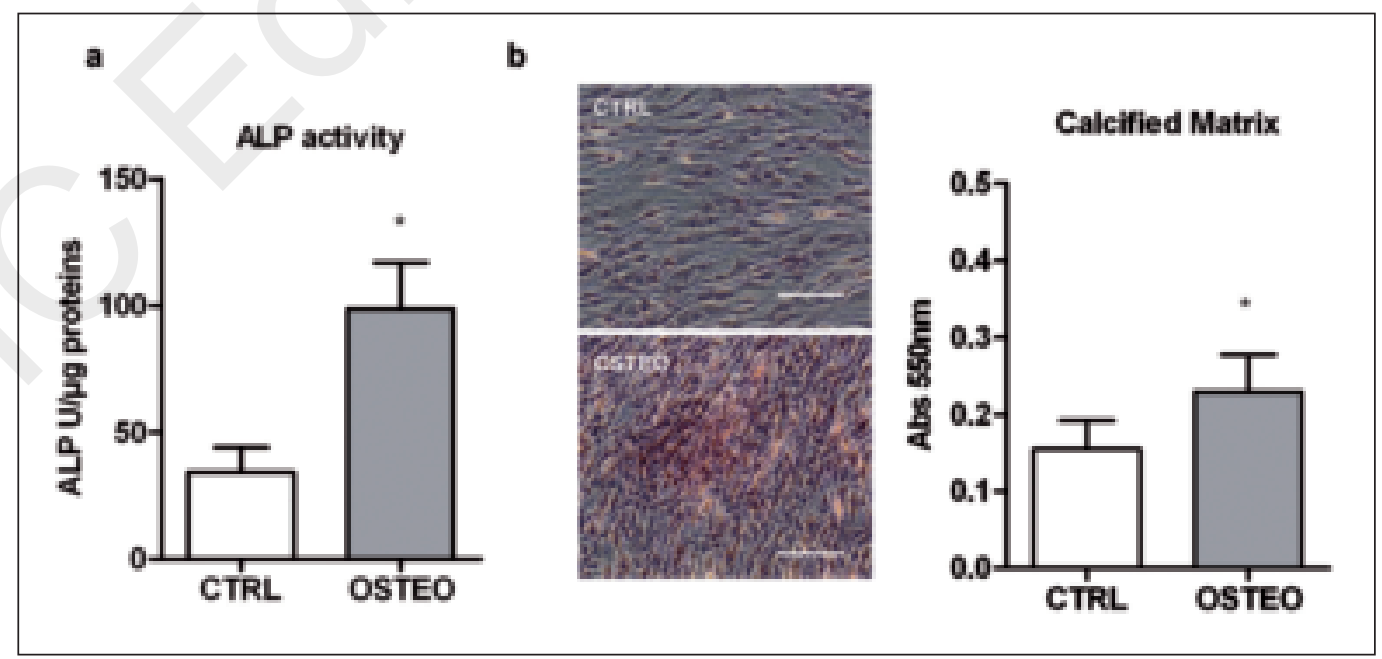

Fig. 5. a: Alkaline phosphatase activity of TSPCs cultured in control (CTRL) or in osteogenic (OSTEO) medium for 14 days. The values were normalized for the total protein content; $b$ : Calcified matrix formation of a representative TSPC population cultured in control (CTRL) or in osteogenic (OSTEO) medium for 21 days and stained with Alizarin Red S (scale bar $=200 \mu \mathrm{m})$. Quantification of the Alizarin Red S dye extraction from both control and osteogenic TSPCs. All data are expressed as mean values \pm standard deviation $(n=3) ;{ }^{*} p<0.05$ OSTEO vS CTRL. 
a

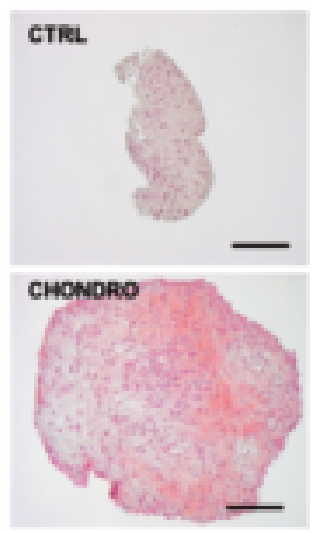

b

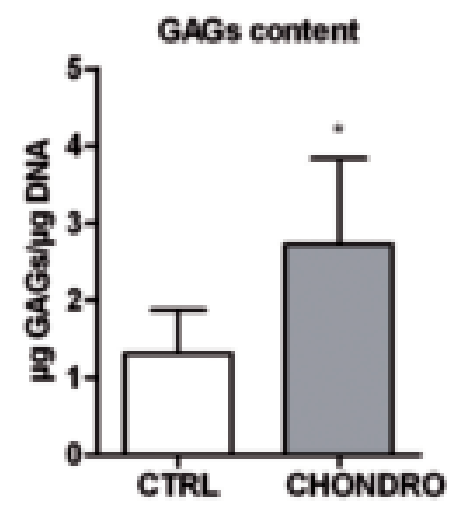

Fig. 6. a: Histological section of a representative TSPC population cultured in pellet form in control (CTRL) or chondrogenic (CHONDRO) medium for 21 days and stained with Safranin O (Scale bar $=200 \mu \mathrm{m}$ ). b: Glycosaminoglycan content (normalized for DNA) assessed by dimethylmethylene blue assay in control or chondrogenic for DNA) assessed by dimethylmethylene blue assay in control or chondrogenic
TSPCs cultured for $\mathbf{2 1}$ days. All data are expressed as mean values \pm standard deviation $(n=3)$; * $p<0.05$ CHONDRO vs CTRL.

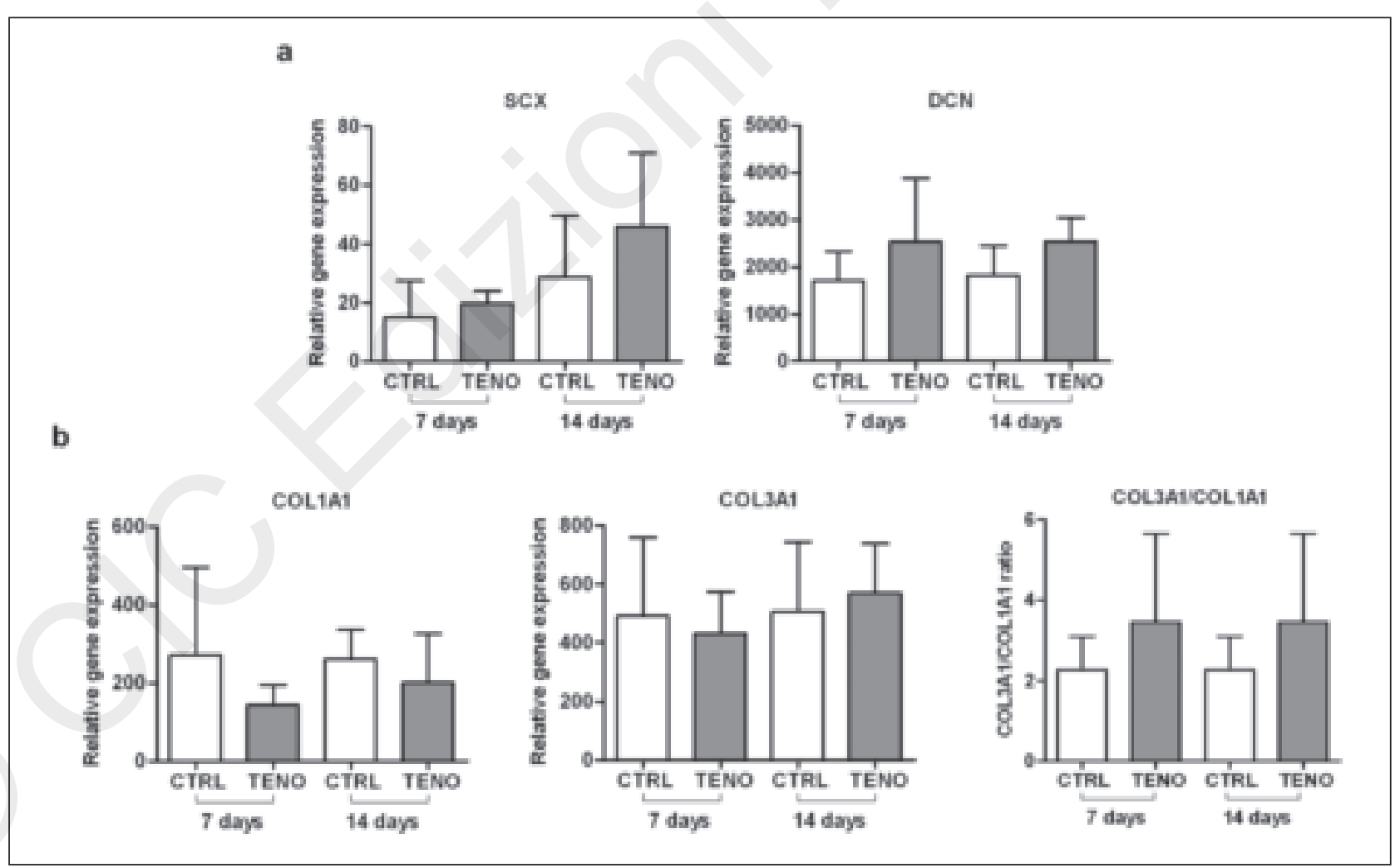

Fig. 7. a: Scleraxis and decorin gene expression of TSPCs cultured for 7 and 14 days in both control (CTRL) and tenogenic (TENO) medium, normalized for housekeeping gene glyceraldehyde-3-phosphate dehydrogenase (GAPDH) expression. b: Collagen type I (COL1A1) and collagen type III (COL3A1) gene expression of TSPCs cultured for 7 and 14 days in both control (CTRL) and tenogenic (TENO) medium, normalized for housekeeping gene glyceraldehyde-3-phosphate dehydrogenase (GAPDH) expression. The ratio between collagen type III and type I is also shown. All data are expressed as mean values \pm standard deviation $(n=3)$. 


\section{Discussion}

Despite advances in the treatment of tendinopathies, several aspects of tendon pathophysiology, which is complex, remain unclear (15). In particular the debate over the mechanism responsible for the activation of the processes that gradually produce degenerative changes in tendon structure and that results in qualitative and quantitative alteration of tenocytes is still open. The discovery of a sub-population of progenitor cells among the tendon cell populations (16) has opened up new perspectives for understanding of tendon physiology and pathophysiology, but above all it has opened new potential doors on alternative approaches for the treatment of tendon disorders. Indeed, TSPCs are supposed to play a primary role in maintaining tissue homeostasis and in promoting repair after injury and thus they could be addressed as a new potential therapeutic target, as well as used for regenerative purposes. In recent decades MSCs have been extensively studied for their ability to differentiate into different cell lineages of mesodermal origin, including bone- and cartilage-like cells that are of particular interest for the orthopedic surgeon (6). Thanks to this property and to other features, such as their simple availability, isolation and expansion procedures, they have been considered to be among the most suitable cell sources for use in regenerative medicine and tissue engineering approaches. MSCs have often been tested in association with several types of scaffold; together they constitute a biological construct that can be surgically implanted in the lesion site. Several scaffolds have been developed and tested for cartilage and bone regeneration, whereas for tendon and ligament reconstruction far fewer attempts have been made. Synthetic polymers and natural ECM components including silk fibroin, poly $(\varepsilon$-caprolactone) (PCL), PCL/poly(DL-lactide) (PLA), poly(DL-lactide-co-glycolide) (PLGA), poly-glycolic acid (PGA) and polysaccharides such as chitosan or various collagen derivates, seem to be the most promising scaffolds for this purpose (17-19). Decellularized tendon matrix has also been investigated as natural scaffold for tendon regeneration $(20,21)$. But all these scaffolds have resulted in neo-tendons or ligaments with lower biomechanical properties in comparison with native ones. Thus, the use of cells in association with scaffolds has been seen as a possible tool to ameliorate the outcome of these regenerative approaches, as cells could help to produce tendon-specific ECM. Moreover, it has been demonstrated that MSCs are able to home to sites of inflammation or tissue injury and secrete massive levels of both immunomodulatory and trophic agents. This means that their therapeutic capacities are not only limited to the ability of MSCs to differentiate into the different end-stage mesenchymal cell types, as traditionally thought. Indeed, more recently their therapeutic effect has been attributed, in particular, to their paracrine function, exerted through the secretion of a broad range of immunomodulatory, angiogenetic, chemoattractive, anti-inflammatory, anti-scarring and antiapoptosis molecules, together with an effect of growth and differentiation support provided by local progenitor cells $(22,23)$. This evidence allows a significant increase in the range of therapeutic applications of MSCs, including the treatment of tendon disorders, where the release of pro-healing factors could contribute to the achievement of satisfactory results. Moreover, due to their low immunogenicity - although this is yet to be studied in TSPCs -, MSCs may be used for allogenic applications, where they may play a role in the process of tendon regeneration, stabilizing or reducing the degeneration of the tendon fibers (24).

Although MSCs from adipose tissue and bone marrow have already been well characterized, little is known about their in vitro tenogenic potential (25). The few findings deriving from animal models showed positive effects of MSCs on tendon repair $(17,26,27)$. In view of this, the aim of our study was to assess the progenitor cell population resident in tendons, in order to propose their possible use in regenerative medicine and tissue engineering approaches. In autologous applications the advantage of this cell source would lie in the prevention of further donor site morbidity, whereas in case of allogenic use TSPCs could be isolated from surgical waste material.

As shown by our data, TSPCs can be isolated from very small fragments of semitendinosus and gracilis tendons and can efficiently proliferate and differentiate in standard culture during passages in culture. Indeed, as shown by the CFU-F, as well as by the immunophenotype analysis, the cell population, initially non-homogeneous, was almost completely homogeneous at passage 4 and presenting mesenchymal-specific features, thus confirming that tenocytes are not able to maintain their phenotype in culture for 
long periods. Both the clonogenic capacity and the immunophenotype of these cells was comparable to that of other MSCs (12): all the MSC-specific markers were highly expressed and, on the other hand, the hematopoietic-specific ones were absent.

In the presence of the adipogenic medium, which was previously developed for adipogenic induction of adipose-derived stem cells (4), TSPCs did not show a marked ability to differentiate into the adipogenic lineage. On the other hand, when exposed to osteogenic stimuli, a noteworthy ability to differentiate into osteoblast-like cells was observed, as revealed by the significantly higher values of both ALP and calcified matrix production compared to the values for control cells. Very surprisingly, TSPCs also possessed a remarkable chondrogenic potential, as shown by the significant increase in GAGs, a typical marker of cartilagelike cells.

Conversely, pre-commitment of TSPCs toward the tenogenic lineage was not observed; indeed, after the tenogenic induction treatment the cells, compared with control cells, were not able to express higher levels of the tendon-specific markers. Nevertheless, it is possible that the levels of scleraxis and decorin, as well as of type I collagen, were already high in the control cells, and this could have partially concealed the potential effect of the treatment on upregulation of these markers. Another possible explanation concerns the tenogenic medium composition; as tenogenic induction has been not adequately investigated yet, other tests should probably be performed to find a better-performing induction medium. Indeed, the use of bone morphogenic proteins (BMPs) such as BMP-12, BMP-13 and BMP-14, members of the transforming growth factor- $\beta$ (TGF- $\beta$ ) gene superfamily, has been found to induce the differentiation of MSCs toward tendon-like cells, as these factors are able promote the ECM synthesis typical of connective tissue. However, different and sometimes conflicting medium compositions containing BMPs can be found in literature, thus indicating that the most efficient one has still to be found. Moreover, while several markers indicating osteogenesis and chondrogenesis processes have been found and accepted by the scientific community, tendon-specific markers remain to be properly identified. In particular, no reliable histological or biochemical assays to detect tenogenic differentiation have been developed, and thus gene expression or protein expression are still the tools most used for this purpose. However, these evaluations are time consuming and expensive, thus preventing the testing of the many possible combinations of growth factors or of other culture parameters that could ultimately identify the most efficient ones.

The limitations of this study include the small number of cell donors, as the large inter-donor variability could have affected the average results. Moreover, a direct comparison with another source of MSCs could have provided more information on their real stemlike phenotype and in particular on their tenogenic potential.

In conclusion, this study showed that human hamstring tendons contain a sub-population of TSPCs that are able to grow in cell culture and show the typical features of MSCs, such as specific MSC marker expression, clonogenic ability and multi-differentiation potential, above all toward osteogenic and chondrogenic lineages. Although the clinical relevance of this study is not direct, it should be considered as one of the first attempts to clarify the biology of tendon cell populations, focusing in particular on the potential applicability of this cell source for regenerative medicine purposes, both in surgical or in conservative treatments for tendon disorders.

\section{References}

1. Sharma P, Maffulli N. Tendinopathy and tendon injury: the future. Disabil Rehabil. 2008;30:1733-1745.

2. Voleti PB, Buckley MR, Soslowsky LJ. Tendon healing: repair and regeneration. Annu Rev Biomed Eng. 2012; 14:47-71.

3. Pittenger MF, Mackay AM, Beck SC, et al. Multilineage potential of adult human mesenchymal stem cells. Science. 1999;284:143-147.

4. de Girolamo L, Lopa S, Arrigoni E, et al. Human adiposederived stem cells isolated from young and elderly women: their differentiation potential and scaffold interaction during in vitro osteoblastic differentiation. Cytotherapy. 2009;11:793-803.

5. Arrigoni E, Lopa S, de Girolamo L, et al. Isolation, characterization and osteogenic differentiation of adipose-derived stem cells: from small to large animal models. Cell Tissue Res. 2009;338:401-411.

6. Caplan AI. Adult mesenchymal stem cells for tissue engineering versus regenerative medicine. J Cell Physiol. 2007;213: 341-347.

7. Rui YF, Lui PP, Li G, et al. Isolation and characterization of multipotent rat tendon-derived stem cells. Tissue Eng Part A. $2010 ; 16: 1549-1558$. 
8. Randelli P, Conforti E, Piccoli M, et al. Isolation and characterization of 2 new human rotator cuff and long head of biceps tendon cells possessing stem cell-like self-renewal and multipotential differentiation capacity. Am J Sports Med. 2013;41:1653-1664.

9. Caplan AI. Review: mesenchymal stem cells: cell-based reconstructive therapy in orthopedics. Tissue Eng. 2005;11:1198-1211.

10. Lopa S, Colombini A, Stanco D, et al. Donor-matched mesenchymal stem cells from knee infrapatellar and subcutaneous adipose tissue of osteoarthritic donors display differential chondrogenic and osteogenic commitment. Eur Cell Mater. 2014;27:298-311.

11. Castro-Malaspina H, Gay RE, Resnick G, et al. Characterization of human bone marrow fibroblast colonyforming cells (CFU-F) and their progeny. Blood. 1980;56:289-301.

12. de Girolamo L, Stanco D, Salvatori L, et al. Stemness and osteogenic and adipogenic potential are differently impaired in subcutaneous and visceral adipose derived stem cells (ASCs) isolated from obese donors. Int J Immunopathol Pharmacol. 2013;26:11-21.

13. Barbero P, Verdun E, Bergui M, et al. High-dose, frequently administered interferon beta therapy for relapsing-remitting multiple sclerosis must be maintained over the long term: the interferon beta dose-reduction study. J Neurol Sci. 2004;222:13-19.

14. de Girolamo L, Stanco D, Galliera E, et al. Low frequency pulsed electromagnetic field affects proliferation, tissue-specific gene expression, and cytokines release of human tendon cells. Cell Biochem Biophys. 2013; 66: 697-708.

15. Del Buono A, Battery L, Denaro V, et al. Tendinopathy and inflammation: some truths. Int J Immunopathol Pharmacol. 2011;24:45-50.

16. Bi Y, Ehirchiou D, Kilts TM, et al. Identification of tendon stem/progenitor cells and the role of the extracellular matrix in their niche. Nat Med. 2007;13:1219-1227.

17. Ouyang HW, Goh JC, Mo XM, et al. The efficacy of bone marrow stromal cell-seeded knitted PLGA fiber scaffold for
Achilles tendon repair. Ann N Y Acad Sci. 2002;961:126129.

18. Lu HH, Cooper JA Jr, Manuel S, et al. Anterior cruciate ligament regeneration using braided biodegradable scaffolds: in vitro optimization studies. Biomaterials. 2005;26: 48054816.

19. Cooper JA, Lu HH, Ko FK, et al. Fiber-based tissue-engineered scaffold for ligament replacement: design considerations and in vitro evaluation. Biomaterials. 2005;26:15231532.

20. Schulze-Tanzil G, Al-Sadi O, Ertel W, et al. Decellularized tendon extracellular matrix-a valuable approach for tendon reconstruction? Cells. 2012;1:1010-1028.

21. Cheng CW, Solorio LD, Alsberg E. Decellularized tissue and cell-derived extracellular matrices as scaffolds for orthopaedic tissue engineering. Biotechnol Adv. 2014;32:462484 .

22. Meirelles Lda S, Fontes AM, Covas DT, et al. Mechanisms involved in the therapeutic properties of mesenchymal stem cells. Cytokine Growth Factor Rev. 2009;20:419-427.

23. Horwitz EM, Le Blanc K, Dominici M, et al. Clarification of the nomenclature for MSC: The International Society for Cellular Therapy position statement. Cytotherapy. 2005;7:393-395.

24. Nixon AJ, Dahlgren LA, Haupt JL, et al. Effect of adiposederived nucleated cell fractions on tendon repair in horses with collagenase-induced tendinitis. Am J Vet Res. 2008;69:928-937.

25. Shen H, Gelberman RH, Silva MJ, et al. BMP12 induces tenogenic differentiation of adipose-derived stromal cells. PLoS One. 2013;8:e77613.

26. Awad HA, Butler DL, Boivin GP, et al. Autologous mesenchymal stem cell-mediated repair of tendon. Tissue Eng. 1999;5:267-277.

27. Chong AK, Ang AD, Goh JC, et al. Bone marrow-derived mesenchymal stem cells influence early tendon-healing in a rabbit achilles tendon model. J Bone Joint Surg Am. 2007;89:74-81. 